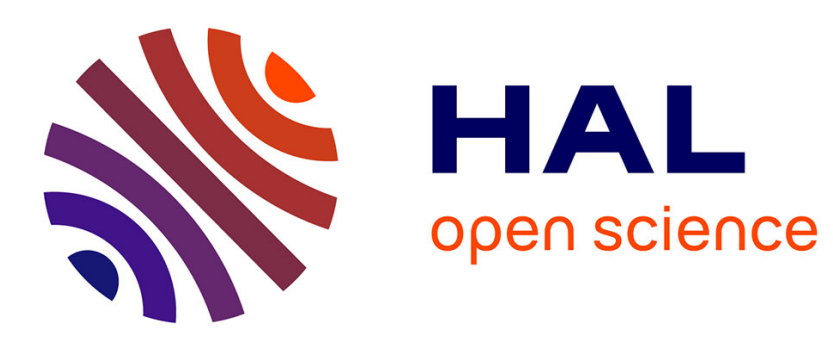

\title{
A multi-agents based E-maintenance system with case-based reasoning decision support
}

\author{
Ren Yu, Benoît Iung, Hervé Panetto
}

\section{To cite this version:}

Ren Yu, Benoît Iung, Hervé Panetto. A multi-agents based E-maintenance system with case-based reasoning decision support. Engineering Applications of Artificial Intelligence, 2003, 16 (4), pp.321333. 10.1016/S0952-1976(03)00079-4 . hal-02162318

\section{HAL Id: hal-02162318 \\ https://hal.science/hal-02162318}

Submitted on 24 Jun 2019

HAL is a multi-disciplinary open access archive for the deposit and dissemination of scientific research documents, whether they are published or not. The documents may come from teaching and research institutions in France or abroad, or from public or private research centers.
L'archive ouverte pluridisciplinaire HAL, est destinée au dépôt et à la diffusion de documents scientifiques de niveau recherche, publiés ou non, émanant des établissements d'enseignement et de recherche français ou étrangers, des laboratoires publics ou privés. 


\title{
A MAS BASED E-MAINTENANCE SYSTEM WITH CBR DECISION SUPPORT
}

\author{
Ren YU ${ }^{1,2}$, Benoit IUNG ${ }^{1}$, Hervé PANETTO ${ }^{1}$ \\ 1: Université de Lorraine, CNRS, CRAN - Faculté des Sciences -BP 23954506 VANDOEUVRE, FRANCE \\ yurenhl@hotmail.com [benoit.iung, herve.panetto@univ-lorraine.fr] \\ 2: HUAZHONG University of Science and Technology - Faculty of Hydroelectric Power - WUHAN, CHINA \\ lqye@hust.edu.cn
}

\begin{abstract}
Today, one challenge of a manufacturer is to maintain with the consumer, the expected service of the supplied product during the whole product life cycle, no matter where the product and the consumer are located. The combination of modern information processing and communication tools, commonly referred to as Tele-service, offers the technical support required to implement this remote service maintenance. However, this technical support is insufficient to face new remote maintenance decision-makings which requires not only informational exchanges between customers and suppliers but also co-operation and negotiation based on the sharing of different complementary and/or contradictory knowledge. It requires an evolution from Tele-service to E-service and e-Maintenance in particular where the maintenance decision-making results from collaboration of maintenance processes and experts to form a DAI environment. For this purpose, a Problem-Oriented Multi-Agent-Based E-Service System (POMAESS) is introduced in this paper. The protocol of negotiation for multi agents and the CBR-based decision support function within this system are discussed, emphasised at the service maintenance problem solving. A prototype system based on these methodologies is developed to demonstrate the feasibility.
\end{abstract}

Key words: Maintenance, E-Service, E-maintenance, Negotiation Protocol, Case Base Reasoning, Multi Agent Systems

\section{INTRODUCTION}

Nowadays, as the competition in global market becomes more and more drastic, the traditional seller market, which mainly delivers products, has been transformed to buyer market, which not only requires products but also emphasises more on solution delivery in regard to user needs. So, one challenge today of industrial enterprise is to maintain with its consumers, the expected service of its product during the whole product life cycle, no matter where the product is located (Schilling, 2000). Indeed, customers do not only expect a very high quality of product from the manufacturer but also a very high quality and effective service (Küssel, et al., 2000).

That means, to operate the maintenance of its equipment out of the hands of the users, sale its service with the products, and achieve near-zero-downtime ability to its products and systems (Koc and Lee, 2002). In the way of maintaining the object service by remote action, the concepts and technique of Tele-service, and more advance, e-service and Tele-service engineering (Lee, 1998), have been developed and applied in industrial processes (machine manufacture, nuclear power plants, robotics), as well as in biomedicine ... The objective of E-Service is to provide the industrial enterprise with abilities of behaviour assessment and performance degradation evaluation, knowledge learning and system failure recovery, and collaborative maintenance to their process and product so as to provide high quality, low cost, timely responsive, and globally available service to customers, normally under some kinds of constraint and in a remote way. (Küssel, et al., 2000) proposes to characterise Tele-service (vs. E-service) using three main criteria:

- Geographical distance between the customer and the supplier. It implies that a supplier who is spatially separated from the customer provides the service.

- Use of information technology required carrying out the service in terms of remote information processing, storing and communication.

- Industrial service. The services have to be in the field of industrial services (maintenance, diagnosis, ...).

In the context of hydropower plant, the application of Tele-service concept was also the objective of the ESPRIT IV-REMAFEX project n'20874 (REmote Maintenance for Facility EXploitation) (Léger, et. al., 1999). It led to the development of a TEMIIS ${ }^{1}$ platform and then of a CASIP $^{2}$ (Computer Aided Safety and Industrial Productivity)

${ }^{1}$ SCHLUMBERGER - SEMA Division Energie - 94126 Fontenay sous Bois (France)

${ }^{2}$ PREDICT - 1 rue carnot - BP $305-54515$ Vandoeuvre (France) (http://www/predict.fr). 
platform for industrial remote maintenance and covering three domains of actions: continuous monitoring of the component degradation and failure, aiding in diagnosis on the basis of localisation and identification processes, providing "tracability" of all the information and actions. It is based on multi layer networks, a local real-time maintenance system (LMS), a centralised maintenance system (with Oracle) and some remote stations. These innovative, generic and open Tele-maintenance platforms (and its additional engineering suite for CASIP) are connected, in CRAN laboratory, to a distributed industrial process regulating a water level in a closed control loop. The loop keeps a constant level (volume) of water (upstream tank) where the input water flow represents the disturbance (recycling pump from the downstream tank), and the valves maintain the level by controlling the output water flow. The "intelligent" valves (Petin, et al., 1998) communicate, from an informational point of view, with the pump, the control and technical management units, and also with the sensors by means of 2 heterogeneous field buses WORLDFIP and Profibus. The Ethernet structure connected to the units and field buses allows remote access to support and integrate Pro-Active Maintenance researches (Iung and Morel, 2000) in the framework of industrial partnership (Figure 1). The main idea is to develop the concept of IMS in terms of Intelligent Manufacturing System vs. Intelligent Maintenance System not only at the Shop Floor level as already tested by (Iung, et al., 1999) but also both at Business and Shop Floor levels. It means to improve the predictive capacities implemented in the Tele-maintenance platform by setting up a new research action as synthesised in (Iung, 2003). This research is completed within both a Chinese-European (HUST and CRAN-UHP) and industrial-academic co-operation sponsored by the European Community and initiated during the INCO-DC EIAM-IPE project $n^{\circ} 961744$. The action aim is to better monitor and anticipate the industrial process degradation and failures by developing an evolution of the platform towards a new one supporting e-maintenance. E-maintenance provides manufacturing and operating systems with near-zero downtime performance through use and integration of $(a)$ real time and smart monitoring, $(b)$ performance assessment methods, and (c) tether-free technologies (Koc et al., 2002). The main issue of this evolution is, for example in relation to the ON/OFF valve component of the industrial process : "How from a degraded situation of the valve, the experts with different domains knowledge and settling in different remote sites can propose to the operator on site (aid of decision making), the best action to do to keep the service of the valve (to forecast the future operational situation of the valve)?" (Iung, 2003).

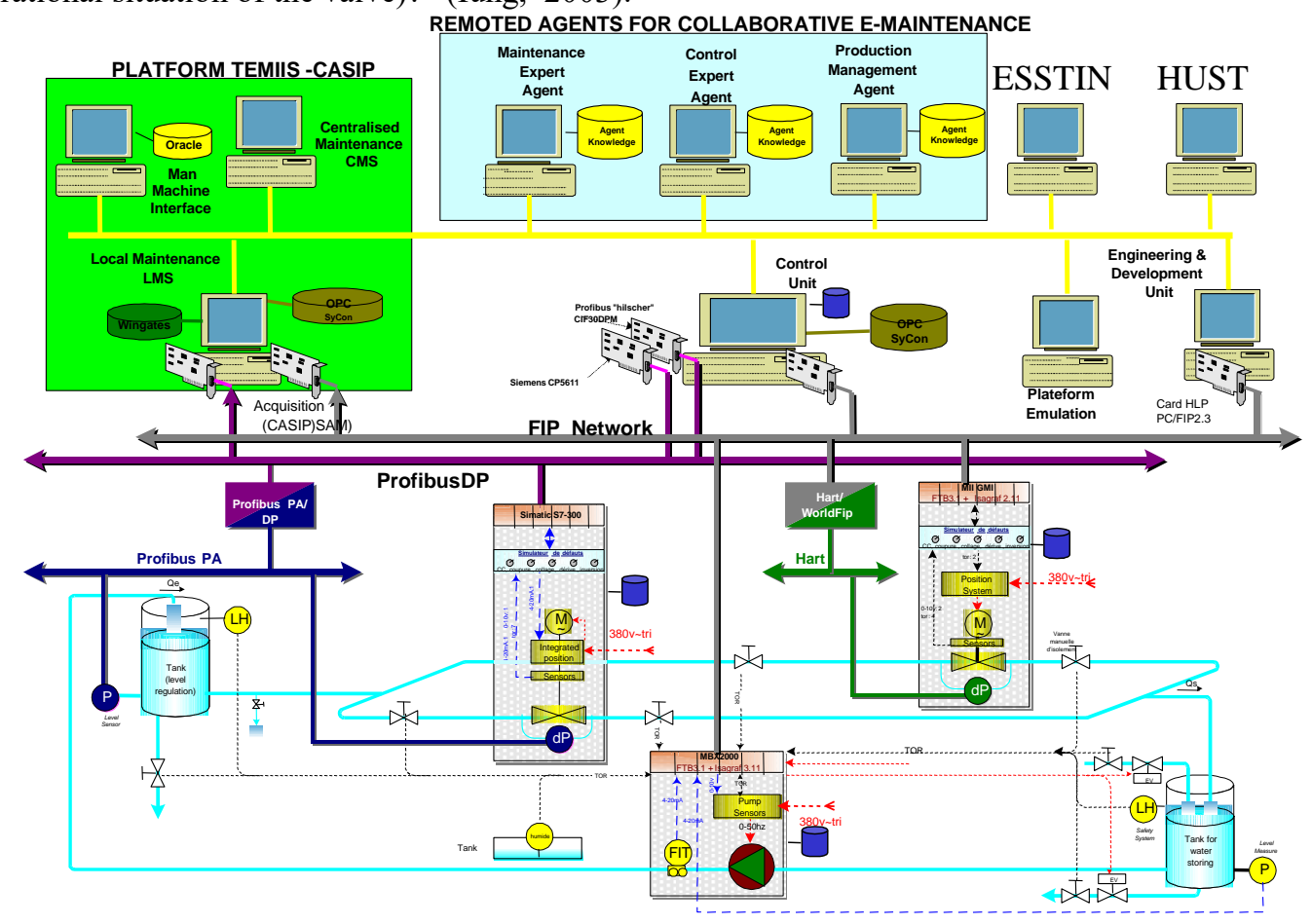

Figure 1: The structure of the platform IMS at CRAN

In that way, the paper developed the concepts of Tele-service and implementation of an e-maintenance platform built on e-service principle. It is based on Multi-Agent System (MAS) paradigm, and named as Problem-Oriented 
Multi-Agent based E-Service (ES) System (POMAESS), in which each agent is a problem solving expert (human or autonomous machine) with different background knowledge and locating in different sites (section 2 and 3). To improve (a) the autonomous decision-making ability of the agents and (b) the convergence time, a decision support method for problem solving related to Case-Based Reasoning (CBR) method (Aamodt and Plaza, 1994), is also integrated to POMAESS (section 4). A prototype is designed and introduced in section 5 in order to assess the feasibility of the methodology of POMAESS and the efficiency of the technologies for realising it. The results are analysed and some future works related to POMAESS are foreseen in section 6.

\section{THE PURPOSE OF POMAESS}

The precondition of giving a good decision to a problem is the ability to get as much knowledge about the problem as possible from different points of view. This needs the coordination, cooperation and negotiation between experts (or agents) representative of these domains. The general definition of an agent is that it's a component of software and/or hardware that is capable of acting exactingly in order to accomplish tasks on behalf of its user (Hyacinth, 1996).

So, the main purpose of POMAESS is to create a system that "interconnects" separated agents (reactive and cognitive agents), by given to them the possibility to collaborate with each other in open and time-constrained environment, thus enabling the whole to operate beyond the capabilities of any of its members, to solve problems within industrial process. The whole behaviour emerges through the dynamics of the interactions of the basic manufacturing agents within the manufacturing environment This synergy is an emergent property of the system as a whole, and it is not expected to be obtained as a simple sum of its components (Valckenaers and al., 2002). In that way, (Shmidt, 1994) defines co-operative work as a work that "emerges when several actors engaged in the fulfilment of a common task, are mutually dependent in their work and have to co-ordinate and collect their individual productions to be capable of making the task which was confided to them ".

It means for the e-service issue related to the CRAN platform, to provide separate experts with a reasonable environment, so that from a degraded situation of "the ON/OFF valve", they can co-operate and make emerging, by negotiation, a solution (the best action) to propose to the operator on site, to solve the problem and keep the service of the valve. This solution is the best right decision to be taken by the operator at the shop floor level to be consistent with the Enterprise strategy level materialised through the expert strategies.

On these basis and as our main objective is to industrially implement and evaluate a collaborative maintenance with the possibility to demonstrate in site its added value, the first POMAESS prototype involves 3 types experts trying to remotely maintain the quality of service of the valve in accordance with the performances strategy.

These three experts are:

- The production management expert whose role to make sure that the water volume to be regulated and fixed by an order will be respected. It can impose on the operator on site a decision to operate the valve in a forced mode to continue production (parameter modification).

- The maintenance expert whose role is to optimise the valve and platform availability by minimising direct and indirect costs related to maintenance. It can impose on the operator on site a decision to stop the valve, to perform a maintenance action on it. With regard to the objective of e-maintenance, one of the contributions is to further formalise the internal behaviour of this expert to help him to propose a decision related to the valve issue.

- The control expert whose role is to ensure that the global functioning of the installation is coherent with regard to the production strategy in order to guarantee that the valve behaviour is consistent with the functioning of the IMS platform. It can impose on the operator on site a decision to do nothing or to restore initial parameters.

The co-operation between these actors can be represented as a multi-agent system because it is defined as a collection of three entities or agents having their own problem-solving capabilities and which are able to interact in order to reach an overall goal (Oliveira, et al., 1999).

The first assumption is therefore that the number of agents is usually small (less than a dozen agents), they are self-motivated (act according to their role) but they have also to join together to work toward a joint goal : to keep the quality of service of the valve (increasing the "benefits" of the valve) even if their role can be sometime contradictory (different priorities, motivations, perceptions).

To lead to the joint goal, our negotiation context corresponds, in relation to the negotiation situations described by (Kraus, et al., 1995), to "Three agents (more than two) who need to share a resource (the valve can be in maintenance mode, in normal control mode, in forced one, ...). One agent has already "access" to the resource. The agents do not have complete information about each other and can unilaterally leave the negotiations". 
With regard to this common objective, the agents have to study the valve degraded situation to propose a future consensual situation, but - on any account - they manage situations where intervenes the notion of operational safety related to the valve or to its role within the platform. Indeed, this safety is directly processed in the intelligent field component itself to be reactive in a very short time (local at shop floor level).

Nevertheless, the valve real time operation implies to take into account for the negotiation limits, the following functional constraints :

- The complete movement of the valve takes about 4 seconds

- The valve in its functioning mode as "modulating" is rather operated for an opening from 0 to $50 \%$ (in $2 \mathrm{~s}$ ). The functioning of this valve is complementary to the modulating valve operating on the second water circuit.

- The external maximum disturbance (unpredictable disturbance) on the input water flow in the main tank is about 5 litres per second (This disturbance is generated by the pump). The tank can be filled totally within 30 seconds (from 0 to $100 \%$ ).

- The valve in a normal functioning of the platform is used about all the 7 seconds (according to the disturbance and to the functioning of the modulating valve).

... completed by some operational constraints (delays, computer time management, ...) such as :

- The time required by the PLC of the valve to generate the vector of degraded situation (20ms),

- The time required to transfer the previous vector on the WordFIP network $(1 \mathrm{Mb} / \mathrm{s})$ to blackboard on the LMS,

- The time required by the agents via Ethernet to access to vector of degraded situation stored in the blackboard,

- The time required by the agents to set-up a proposal (to select an alternative),

- $\quad$ The time required by the agents to exchange the proposal on the Ethernet network $(100 \mathrm{Mb} / \mathrm{s}), \ldots$

These functional and operational constraints which underline a global time constraint in terms of seconds (and not minutes or hours), implies first, that the prototype will be focused on valve "slow" degradation (and not on sudden ones) potentially observable, and then, more fast solution to be achieved will be proposed (convergence to be obtained quiet in minute), faster degradation will be treated and the consequences mastered (no evolution towards the failure, no useless stop, ...).

\section{THE COORDINATION AND NEGOTIATION PROTOCOL IN POMAESS}

On the previous constraints and according to the different patterns of cooperation and negotiation protocols (Paulo, et al., 1999) (Kraus et al., 1995) (Rosenschein, et al., 1994) having their relevant presumptions, constraints, advantages, disadvantages and application domains, the negotiation in POMAESS (Yu et al., 2002) is:

- a kind of collaboration for a common goal, not a kind of competition. On the one hand, all agents would like to try to give out his best proposal on the base of his background knowledge, in order to make his proposal win out. On the other hand, the agents are from different domains (Maintenance, Production, ...). In order to persuade the other agents to agree with his opinion, one agent must try to make, for other agents, his opinion understandable, and defends the reason of his opinion. So, in POMAESS, it is important to let agents to understand and get full information with each other, but not to try to hide his contemplation.

- time constrained due to the industrial application constants (limited time in order to catch up with the change of state of device and with the anticipation decision). The global time of negotiation represented by a set $\tau\{0,1$, $2, \ldots\}$ of t periods (one period per action) is limited by a maximum time related to the time constant of the valve degradation. This time, which is appropriate for every current degradation, represents the maximum temporal interval between the degradation and failure. This time is evaluated according to the return of experience, vendor data, ... By respecting this time, the valve service is preserved as much as possible, and the degraded valve information consumed by the agents on which is based the negotiation corresponds to the real state of the valve (no gap between the physical component state and its informational representation).

- compromise-based because the contents of proposals from different agents will not always be contrast, but have some overlap. The judgement of one agent to others' proposals will not absolutely be "accept" or "reject", but also some medium choice. In most situations, no one agent has a global knowledge to the whole problem in POMAESS, so it's hard to find a mediator. In the mean time, no matter if all the agents can reach a completely receivable agreement, the final decision must be made to treat the problem within a given time.

In order to realise the goal of POMAESS, some countermeasures are adopted to cope with these characteristics and constraints. In order to form a benchmark for meaning expressing and assessing the validity and correctness of 
proposals, some fundamental interests are introduced as the attributes of proposal. Considering the goal of POMAESS for industrial process problem solving, the fundamental interests may mainly lay in four aspects: economic, performance, reliability and availability. These aspects has to ensure the optimisation of the CRAMP parameters (Cost, Reliability, Availability, Maintainability, and Productivity) by keeping also the safety context. A proposal is composed with the content, the evaluation of contribution to interests (ECI), and explanation :

- The content is the description of the decision of an agent about how to deal with the problem (degraded valve) in next step. The initial set of available alternatives proposed to the agents is composed of 5 elements (to stop the valve, to operate in this mode, to change functioning parameters, to change the functioning mode, to launch in parallel a checking of the component).

- The evaluation expresses the forecast of the agent about the contribution of the proposal to interests, and is given with unitary numbers, like 0.8 to economic aspect but 0.4 to reliability ( 1 is the highest level);

- The explanation illustrates why the agent gives this decision and why he thinks the decision influences the different aspects of problem solving like this.

By this way, each proposal from different agents brings with full information related to this agent's knowledge. Each agent is endowed with different weights in different interest aspects according to its background knowledge when the final decision is calculated.

From a technical point of view, the architecture of co-ordination in POMAESS is a combination of master-slaver co-ordination pattern, blackboard structure and committee formation (Winter, 1997). Two kinds of agent are adopted in the system. One is Negotiation Agent (NA), which launches and organises the negotiation, calculates the outcome set based on the proposals of Expert Agent (EA), but never gives his own opinion about the problem solution. It knows well about problems but can know nothing about the problem solving. Another one is Expert Agents, which are invited by the NA according to the need of the problem solution, and organised as a committee. They are experts for the problem solving. NA provides a blackboard and all EAs can get relevant information of the problem from it.

The information stored in the blackboard and related to the informational situation produced with the valve at the degradation time, is composed of :

- valve position,

- valve torque,

- water flow,

- the most likely cause of the degradation (result of the diagnosis already implemented on the valve) (Pétin et al., 1998),

- a global state of the valve degradation (Boolean) materialising the "no degradation state" by the value 0 and "degradation state" by the value 1 .

Then an agent prompts his proposal to all other agents, discusses with other EAs about any proposal, adjusts their own proposal until all the proposals are inclined to be unanimous, or the time of negotiation is up. There is only one NA, which can be acted by any EA, or field operator agent, but many EAs in the system.

NA launches the negotiation only according to the emerging of problem. Every EA can put forward and modifies his own proposal, as well as it gives suggestions about other agents' proposals at any time. A suggestion is represented by completely "receive" or "reject", as well as the modification of the values of ECI of this proposal to the interests. Based on the hypotheses that all the agents are benevolent, trustful, and tolerable with each other, it's possible for EA to overcome the conflicts and overlaps between them, and to reach a commonly accepted outcome after they adjust their opinion according to their own knowledge and the increasing comprehension to each others. EA can also give his explanation about his suggestion and proposal. By this way, he can try to persuade other agents to accept his opinion, as well as each agent can get as excessive as possible of information and knowledge from others to improve their proposal and suggestion as correct and complete as possible.

The co-ordination will be terminated according to the following rules:

Rule 1-1: If all registered agents permit to end the coordination;

Rule 1-2: If the time of coordination is up.

NA checks if there is any equilibrium proposal achieved. The rules to judge if a proposal can be an outcome, are:

Rule 2-1: If all agents registered in the negotiation accept a proposal, it can be an outcome;

Rule 2-2: If the time is up and no proposal is completely accepted by all agents, NA needs to calculate the outcome according to the arithmetic described below: 
To a particular proposal $P_{i}(i=1,2, \ldots, m)$, the opinion of the $E C I$ value of the agent $A_{j}(j=1,2, \ldots, n)$ to the interest $I_{k}$ $(k=1,2, \ldots, r)$ is $O_{j \rightarrow i, k}$. The averages of different agent's ECI of the interest $I_{k}$ to proposal $P_{i}$ is $S_{i, k}$, are calculated as:

$$
S_{i, k}=\frac{1}{n} \sum_{j=1}^{n} w_{j, k} O_{j \rightarrow i, k}
$$

in which: $w_{j, k}$ is the weight of interest $I_{k}$ in the point of view of the agent $A_{j}$. For example, maintenance agent has higher weight than Control Agent in reliability and availability interests, but lower in performance interest.

By the end of time, if some agents never give any opinion or response to some proposal, when the $\mathrm{S}_{\mathrm{i}, \mathrm{k}}$ to these proposal are calculated, these agents need to be excluded.

Then, to the proposal $\mathrm{P}_{\mathrm{i}}$, the final criterion $\mathrm{C}_{\mathrm{i}}$ can by calculated as:

$$
C_{i}=\sum_{k=1}^{r} w_{k} S_{i, k}
$$

in which: $w_{k}$ is the weight of the interest $I_{k}$ to the final criterion. For example, when the maintenance issue is considered, the weight of reliability and availability interests will be higher than performance interest.

When all the final criterion of proposals are counted, the proposal with the highest final criterion $D=\max \left\{C_{i} \mid i=1\right.$, $2, \ldots, \mathrm{m}\}$ is chosen as the final decision to the problem. Then the negotiation of this session is terminated.

The application of these rules allows finding solution, which could be learned and reused in other situations. Indeed to ensure a faster convergence of negotiation and to improve the autonomous decision-making ability of the agents within the e-maintenance platform, a "right" starting point for EAs to negotiate or an initial set of candidate proposal can be developed. This requires the integration of some other AI techniques into the POMAESS. One solution is to deploy CBR techniques to POMAESS.

\section{CBR TECHNIQUE AND ITS INTEGRATION TO POMAESS}

Basically, CBR is a method to solve a new problem by remembering a previous similar situation and by reusing information and knowledge of that situation (Aamodt and Plaza, 1994). Indeed, the foundation principle of CBR is the fact that "Similar problems have similar solutions" (Bergmann and Wilke, 1996). Normally, each case consists of two parts: Problem Description and Solution, and the typical problem-solving cycle of a CBR tool is based on five phases: retrieval, reuse, adaptation, review and storage. CBR has been used to create numerous applications in a wide range of domains, especially in the area of interactive troubleshooting.

So, our purpose is to try to integrate CBR to provide the decision support ability to each expert agent within the POMAESS. Concretely when the valve degradation situation is taken into account (the problem), the support system needs to select some "as if" proper solutions for this situation from past cases, so as to form a reasonable starting point for the negotiation of Eas. It has to improve the accuracy of original proposals and reduce the time for negotiation and problem solving (convergence time).

\subsection{What is a case in POMAESS?}

To describe the status of a dynamic system in industrial process, a series of parameters that can reflect if the function of the system is correctly performed, are usually adopted. For example, for the valve of the platform in CRAN, the torque of the valve, the water flow, the position of the valve can be taken into account. These parameters form the state space or problem space (PS) of a maintenance object. They should be available and sensitive to both functions and faults, and can be continuing value as well as discrete value. Every possible state of the maintenance object described by these parameters is seen as a point in the state space. The dimension of the state space equals to the number of the selected parameters. The state of the maintenance object can be described with a vector represented by a table PST in knowledge base:

$$
P S=\left\{p_{1}, p_{2}, \ldots \ldots, p_{n}\right\} \in R^{n} \quad(n \text { is the number of the selected parameters })
$$

To each state of the maintenance object, there are some different solutions from different interest points of view. For example, from a reliability point of view, the proposal "to stop the valve" may be the best decision but from economic point of view, the best decision may be "to operate in this mode". This is also the reason why the ECI of different interests for each proposal is introduced in the POMAESS. The content of the solution under some constraints is 
usually given in natural language form while the ECI are given with unitary numbers as described before. So, the ECI to a solution forms another space (ES) for each point in PS, and each point in ES has a corresponding content of a solution. The dimension of the EA equals to the number of the selected interests. The ES can be described with another vector: $\quad E S=\left\{e_{1}, e_{2}, \ldots \ldots, e_{m}\right\} \in R^{m} \quad(m$ is the number of the selected interests)

The content of solutions forms a one-dimension space that is named as decision space (DS). Another table DST in knowledge base can represent the ES and DS. The relationship of these spaces is illustrated in the figure 2.

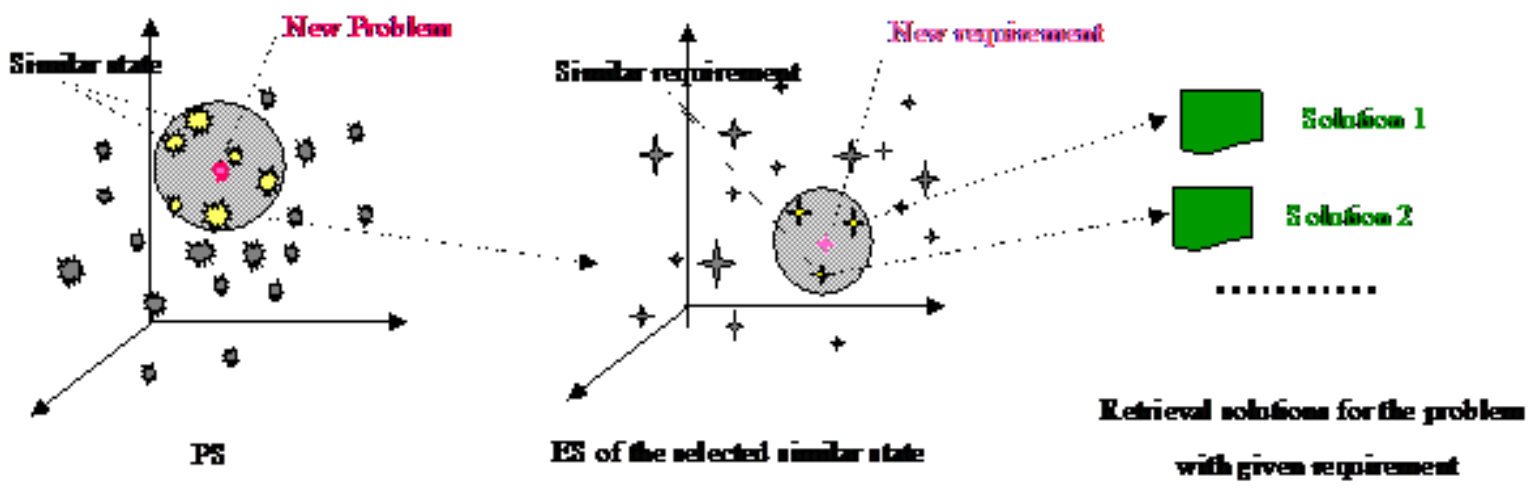

Figure 2: The relationship of the three spaces

Thus, in the decision support system, the case is defined as a set $\mathrm{C}=$ \{process state (PS), decision content (DS), evaluation value set of decision to the interests (ES) \}. A point in PS has a corresponding ES, and each point in this ES has a corresponding point in DS. Finally, the process of case-based reasoning in the decision support system is: find several points in PS which are within a given distance to the current state point of the industrial process; according to the requirement of the interests, retrieval several solutions which are corresponding to the selected points in PS and can satisfy these requirements of interests. The retrieved solutions are provided to each EA as the original solution set for discussing, the EAs can choice one of them as his proposal, as well as modify them, until the final outcome of negotiation is gotten.

\subsection{How to retrieve similar cases?}

Because the industrial processes are usually real-time dynamic processes, so the speed of retrieval is very important. Besides this, the retrieval process must be complete and flexible, and the construction and maintenance of the case-base must be straightforward and easy practised. Normally, to retrieve the similar cases, it requires calculating the "similarity" or "distance" of the new state with all the existing states in case base. To continuous value, like the torque of the valve, the similarity $s_{a}$ of two values $a_{1}$ and $a_{2}$ is defined as:

$$
\mathrm{s}_{\mathrm{a}}=\left|\mathrm{a}_{1}-\mathrm{a}_{2}\right|
$$

To discrete value, like the position of the ON/OFF valve, or the index of a list, as each number represents an absolute state, the similarity $s_{d}$ of two values $d_{1}$ and $d_{2}$ is defined as:

$$
s_{d}= \begin{cases}0 & d_{1}=d_{2} \\ \infty & d_{1} \neq d_{2}\end{cases}
$$

In order to improve the speed of similar case retrieval, the calculation of similarity of the states is combined with the case searching in case base, and some methods are adopted.

To retrieve the similar existing state for a new problem, the PS is considered first. The discrete parameters are invested one by one, and only the cases for which values of the discrete parameters have similarity with the input value (equal to 0 ), will be reserved. Then, the continuous parameters are investigated one by one, the similarities between the input values and those in reserved cases are calculated, and the cases with the similarities within given levels are selected. An important thing here is the choice of the limitation values for selecting cases in PS according to continuous parameters. These limitations represent the weight of parameters. If a parameter is important, the cases that will be selected should have this parameter more similar to the input one, so the limitation value should be lower, and vice versa. 
Unlike the parameters for state describing, the requirement for problem solving is usually a fuzzy concept, like "emphasize the reliability very much but less emphasize the economic". For this reason, to retrieve the cases that have the similar requirement for problem solving (according to the value of ECI), some fuzzy concepts are also integrated. When the ECI of an interest is 0 , it represents that don't care this kind of interest at all. When it is 1 , it represents that this kind of interest is cared very much. Between the interval $[0,1]$, several level stages can be developed, which represent different requirement degrees to this interest. Each stage has an index number.

In ES, the solutions that have the similar interests' degree with the requirement to a state, are retrieved with the following steps: Take the previous selected cases in PS into account one by one, read a requirement of one interest, choice the solutions for the state of the selected case, for which ECI to this interest is near the given requirement. After all the interests are considered, the remained solutions are the results that have the similar state to the problem and can satisfy the requirement of problem solving in a predefined degree.

\subsection{The method of adaptation and feedback of retrieved cases}

The retrieved cases are not always completely and directly suitable to a new problem solving. Normally, their solutions need to be adjusted and modified in order to solve the new problem. Thanks to the negotiation system designed before, this work can be carried out by the negotiation between different expert agents effectively.

In the beginning, the case base is empty but by the analysis of the maintenance object and the experience of the operation, some rules about the problem solving can be drawn. These rules can be expressed like the case defined before. That is expressing the rules with the points in PS, ES, and DS. These points form the original point set in these spaces. During the operation of the system, there will be more and more cases, and the densities of the points in three spaces will be more and more high, so the solutions in these cases will be more and more accurate to the correct solution to the problem. Based on these more and more accurate original solution, the time needed for expert agents to negotiation and find the final solution will be more and more less (to be faster convergent).

\section{THE PROTOTYPE POMAESS}

In order to assess the feasibility of the methodology of POMAESS, a prototype system based on the concept of POMAESS is designed to provide the E-Service ability to the CRAN experimental platform. The service object is the ON/OFF valve of this platform.

The prototype system is developed with the Java language, and Aglet API. Aglets are free mobile agents developing kit developed by IBM (http://www.trl.ibm.com/aglets/) (Danny, et al., 1998). It's a set of JAVA classes and interfaces that allows creating mobile JAVA agents. It mirrors the applet model in JAVA, and can be run in any agent host that supports JAAPI, with platform independence; it can be dispatched as an object, to remote site, as well as retracted from remote site. It can be cloned, deactivated/activated, and disposed.

From communication point of view, an aglet object can carry information and its executing state with itself and move to destination site and resume at there. It also can exchange information with other Aglets in form of message. The message can be any structure of data supported by Java, as well as a JAVA object.

To industrial problem solving, one of important issue in remote distributed application is security as for example those developed between France and China for our experimentation. In the prototype system, the security is ensured in two layers. One is in application layer, in which an original relevant EAs list (OAL) and a dynamic relevant EAs list (DAL) are designed to ensure the security of the problem and the secret and validity of messages. Another one is in implementation layer, which is ensured by the security model of JAVA and Aglet API.

Moreover, in the initialisation period, the prototype has to be configured according to the problem. The following things need to be defined: the necessary kinds of data for the judgement of the status to the field process (for the valve, it has been chosen the variables informing on the torque, the position, ... ); the time limit of the negotiation of the problem; the possible solutions of the problem ("to stop the valve", ...); the initial expert agent list of the problem (including EA name, address, each agent's weights to each interest); the weights of different interests to the problem solving.

From this step and when the IMS platform is running, for each degradation detected ( the Boolean global state of the valve degradation is equal to 1 ; the valve variables are transferred via the WorldFip network to the blackboard implemented in the LMS), the NA begins the process of negotiation (Figure 3) initialising and launches a negotiation. First, it dispatches EAs to proper site according to the OAL, sends relevant information to every existing EA, and forms the DAL according to each agent state and the response about if they want to participate in this negotiation. 
Besides these, the CBR decision support system is launched and some initial proposals that suitable for the similar problem solving are retrieved and scattered to each EA. Then, it can start the negotiation among those registered EAs.

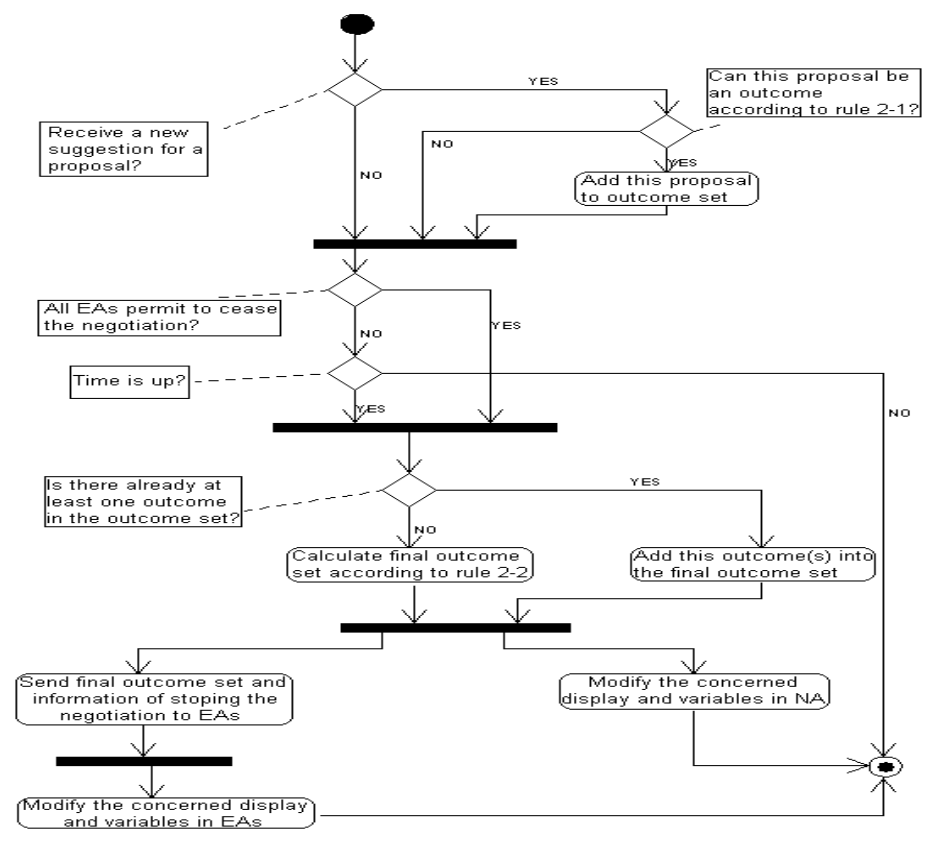

Figure 3. The activity diagram of NA to calculate the final outcome set

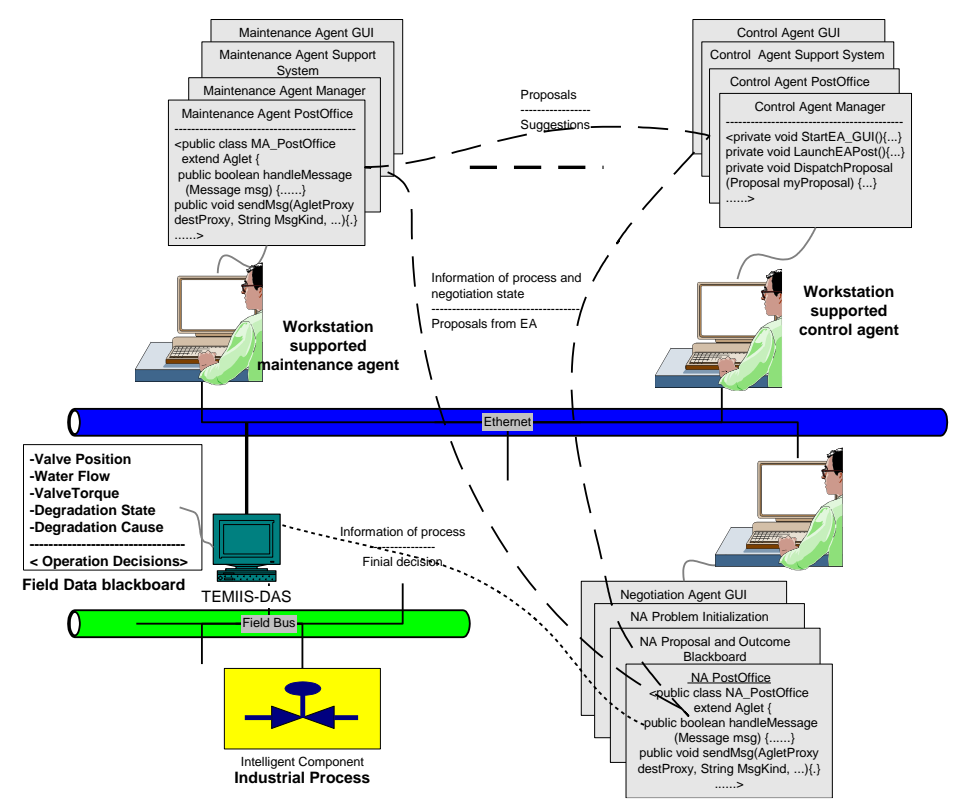

Figure 4: The work manner and the construct of the prototype system between the three agents

During the negotiation, every expert agent can register in or out, as he likes at any time (e.g. the first experimentation 
is made between the three agents). Only after he registers in, he can bring forward his proposals as well as his suggestions to other agents' proposals.

Each agent built the proposal in relation to his knowledge management related to his ability domain. For example, we have experimented the development of the maintenance expert decision-making on the basis of prognostic process from models of knowledge on the functioning-malfunctioning of the system (Léger et al., 1999). He can therefore from an instance of the model (e.g. FMECA representation coupled with Bayesian Network (Weber et al., 2001)) which takes into account the degraded situation of the valve, to go through this model in inverse causality mode to foresee towards which states the system can evolve considering the degradation and then evaluate for each state some maintenance strategies (in relation to the CRAMP parameters) leading to submit one proposal with interest assessment.

At the beginning of the negotiation implementation, the agent can choice one proposal from the initial list and then modify it according his own knowledge and the suggestions from other EAs. All proposals are shown to every agent, one agent can give his proposals at any time to all other agents, and give his suggestions to any others' proposals for discussing (Figure 4). All the suggestions from different agents to a proposal can also be seen to all EAs. The form of proposal and suggestion follows the definition given before with a first use of CBR techniques. As the negotiation lasting, the NA is monitoring the process, and judging if any proposals can be an outcome, as well as if the negotiation can be terminated. As the original proposals are suitable to the similar problem solving, the convergence time of the negotiation can be reduced. At the end, the NA transfers the final outcome into the LMS as a complex variable (content + interest + explanation) and then the LMS put this variable at the disposal of the operator on site (to help him to take the right decision) through the screen of the control unit at the shop floor level.

\section{RESULTS AND FUTURE WORKS}

The POMAESS prototype connected with the real IMS platform (link through the blackboard with the shop floor information) is operational and has been tested on different types of valve degradation (execution from simulated valve degradation of different negotiation scenarios with successful convergence). It allows to a expert wherever he is, to make software configuration in very short way to participate to the negotiation. All the results show that the idea of POMAESS is feasible to provide solution for industrial process problem solving from remote site within a network environment, and the adopted technology for developing the POMAESS is effective. It can be a choice for the solution of high quality but low cost and rapidly responsive services for industrial process. The designed method of the CBR-based decision support system for retrieving similar cases is simple, so, high retrieval speed can be ensured (experimentation in progress). And the retrieved cases can satisfy the different requirement of the problem solving because of the introduction of the ECI of the interests for problem solving.

Nevertheless even if the results are promising from a feasibility and efficiency point of view, the experimentation context is too much restricted (enlarge the assumptions context is needed) to generalise these results especially by guaranteeing that the dynamics of the agents is always coherent and convergent.

So, in future, more concrete work should be done to validate more globally the decision support system. Beside this, the methods to properly set and adjust the limitation values for similar state retrieval according to the need of problem solving and the density of existing cases should be investigated further. Moreover some work has to be planned to formally prove the dynamics of the agent interaction and of the result emergence.

\section{ACKNOWLEDGMENTS}

The context and concepts of this work originated from the ESPRIT REMAFEX project and from Chinese-European co-operation within the INCO-DC EIAM-IPE project $n^{\circ} 961744$. We thank all the partners of these projects for their co-operation and significant contribution. This work is pursued today within the framework of the European ESPRIT V - CENNET project n ${ }^{\circ}$ IST-2000 -28739 (China Europe Network on the Net). 


\section{REFERENCES}

Aamodt, A. and Plaza, E. (1994). Case-Based Reasoning : Foundational Issues, Methodological Variations, and System Approaches. AI Communications. IOS Press, vol. 7 : 1, pp 39-59.

Bergmann, R. and Wilke, W. (1996). On the role of abstraction in case-based reasoning. Third European Workshop on Case-Based Reasoning, Lecture Notes in Artificial Intelligence, Springer Verlag, pp 28-43.

Danny, B. and Mitsuru, O. (1998). Programming and Developing JAVA Mobile Agents with Aglets. ADDISON-WESLEY, ISBN 0-201-32582-9.

Hyacinth, N. (1996). Software Agents: An Overview. Knowledge Engineering Review Journal, vol. 11(3), November. Iung, B., Muhl, E., Léger, J.B., Morel, G. (1999). MAS modelling paradigm application for the development of a proactive maintenance intelligent system. 1st IFAC-MAS'99 Workshop on Multi-Agent-Systems in Production, pp 89-96, December 2-4, Vienna, Austria.

Iung, B. and Morel, G. (2000). Towards Intelligent Maintenance Integrated within the Enterprise. MCPL'2000, 2nd IFAC-IFIP-IEEE Conference on Management and Control of Production and Logistics. Invited Lecture. pp 414-420, 5-8 July, Grenoble, France.

Iung, B. (2003). From Remote Maintenance to MAS-based E-Maintenance of an industrial process. International Journal of Intelligent Manufacturing. Eds A. Kusiak, Special issue on Internet-Based Distributed Intelligent Manufacturing System (Eds E. Banaszak), vol. 14, No. 1., January.

Koc, M. and Lee, J. (2002). A System Framework for Next-Generation E-Maintenance Systems. http://www.uwm.edu/CEAS//ims/pdffiles/E-Maintenance.pdf

Koc, M., Ni, J., Lee, J. (2002). Introduction of e-manufacturing. 5th International Conference on Managing Innovative Manufacturing and E-Business Integration (MIME); September 9-12, 2002, Univ. of Wisconsin Milwaukee, Wisconsin, USA

Kraus, S., Wilkenfeld, J., Zlotkin, G., (1995). Multi-agent negotiation under time constraints. Artificial Intelligence. vol. 75, pp 297-345.

Küssel, R., Liestmann, V., Spiess, M., Stich, V. (2000). "Teleservice” a customer oriented and efficient service. Journal of Material Processing Technology. vol. 107, pp 363-371.

Lee, J. (1998). Teleservice Engineering in Manufacturing: Challenges and Opportunities. International Journal of Machine Tools \& Manufacture, vol. 38, pp 901-910.

Léger, J-B., Iung, B., Ferro Beca, A., Pinoteau, J. (1999). An innovative approach for new Distributed Maintenance System : application to Hydro Power Plants of the REMAFEX project. Computers in Industry, vol. 38, issue 2, pp 133-150, February.

Oliveira, E., Fisher, K., Stepankova, O. (1999). Multi-agent systems: which research for which applications. Robotics and Autonomous Systems. vol. 27, pp 91-106.

Paulo, S. and Carlos, R. (1999). A Distributed Architecture and Negotiation Protocol for Scheduling in Manufacturing Systems. Computers in Industry, vol. 38, Issue 2, pp103-113, March.

Petin , J. F., Iung, B., Morel, G. (1998). Distributed Intelligent Actuation and Measurement (IAM) System within an Integrated Shop-Floor Organization. Computers in Industry. vol. 37. pp197-211.

Rosenschein, J.S. and Zlotkin, G. (1994). Rules of Encounter: Designing Conventions for Automated Negotiation among Computers. MIT Press.

Schilling, K. (2000). Telediagnosis and Teleinspection Potential of Telematic Techniques. Advances in Engineering Software. vol. 31. pp 875-879.

Shmidt K. (1994). Modes and Mechanisms of interaction in co-operative work. Riso, National Laboratory.

Valckenaers, P., Van Brusel H., Hadeli, Bochmann O., Zamiferescu C. On the design of complex emergent systems. IFAC World Congress, July 2002, Barcelona, Spain.

Weber, P., Suhner, M.-C., Iung, B. (2001). System Approach-Based Bayesian Network to aid Maintenance of Manufacturing Process. In: 6th IFAC Symposium on Cost Oriented Automation (Low Cost Automation 2001). October 8-9, Berlin, Germany.

Winter, E. (1997). Negotiations in Multi-Issue Committees. Journal of Public Economics. vol. 65, pp323-342. 1997.

Yu, R., Iung, B., Panetto, H., Ye, L., Morel, G.; A Mas and CBR Based E-maintenance for Prognosis in Industrial Process; 5th International Conference on Managing Innovative Manufacturing and E-Business Integration (MIME); September 9-12, 2002, Univ. of Wisconsin Milwaukee, Wisconsin, USA. 